$16^{\text {th }}$ International Congress of Metrology, 08002 (2013)

DOI: $10.1051 /$ metrology/201308002

C Owned by the authors, published by EDP Sciences, 2013

\title{
Mesurage de perçages imparfaits au défilé par vision industrielle
}

\author{
Fabrice Mairesse $^{1, a}$, Tadeusz Sliwa ${ }^{1}$ et Yvon Voisin ${ }^{1}$ \\ ${ }^{1}$ Laboratoire Le2i - UMR CNRS 6306, BP16, 89010 Auxerre Cedex, France
}

\begin{abstract}
Résumé. Cet article traite de la mesure sur chaînes de production par vision industrielle et plus particulièrement du mesurage de perçages sur panneaux manufacturés. Pour cela, nous nous intéressons au contour de ces usinages à la surface du panneau. Ces formes sont communément considérées, en première approche, comme des cercles ou des ellipses ce qui peut être assez éloigné de la réalité notamment pour des matériaux compressés. D'autres phénomènes tels que l'environnement industriel et la chaîne d'acquisition interfèrent également sur la qualité de l'image obtenue. Afin de répondre à cette problématique, les auteurs présentent une comparaison de différentes méthodes d'estimation de cercles dans le cadre de déformations de formes. Une méthode de correction de formes est proposée dans le but d'améliorer la mesure. Les résultats obtenus montrent une amélioration significative de l'estimation des perçages pour certains estimateurs et notamment dans les cas de perçages de faible diamètre apparent.
\end{abstract}

\begin{abstract}
This article deals with measurement on production lines by industrial vision. More precisely, it concerns the measurement of drillings on manufactured panels. We are interested in the contour of drillings in the panel plan. These forms can be considered, in a first approach, as circles or ellipses that are not true notably in compressed material. Others phenomena such as industrial environment and acquisition chain can also interfere on the image quality. In order to answer this problematic, the authors present a comparison of several measurement methods on distort circles. A method to regularize forms is proposed in order to increase measure quality. The results indicate that the validity of the measure obviously depends on the computation methods but also on the diameter of the drilling.
\end{abstract}

\section{Introduction}

Les domaines de la vision sont nombreux. Ils vont de la vidéosurveillance au contrôle qualité. Ce dernier peut être qualitatif ou quantitatif. Cette étude se concentre sur ce dernier aspect et plus particulièrement sur la mesure en industrie. Le contrôle dimensionnel de biens manufacturés en est un pan complet et peut porter sur de nombreux points: mesure de dimensions globales ou locales, équerrage, mesure de formes : circularité...

Les outils de mesure de formes de base sont désormais accessibles dans toutes les machines de vision. Il est en effet aisé de mesurer une ligne, un carré ou encore un cercle soit automatiquement soit à l'aide d'une interface graphique.

Cependant, ces outils se basent sur des formes mathématiques. Or les formes obtenues par un capteur sont au mieux discrétisées, ce qui peut impliquer une déformation notable dans le cas de formes de petites tailles (Fig. 1), au pire bruitées et déformées. Ceci est notamment le résultat de la chaîne d'acquisition (qualité des composants optiques, bruit électronique, ...) et de l'environnement (poussière, vibration, objet en déplacement, ...).

Figure 1. Cercle discrétisé décalé par pas de 0.125 pixels

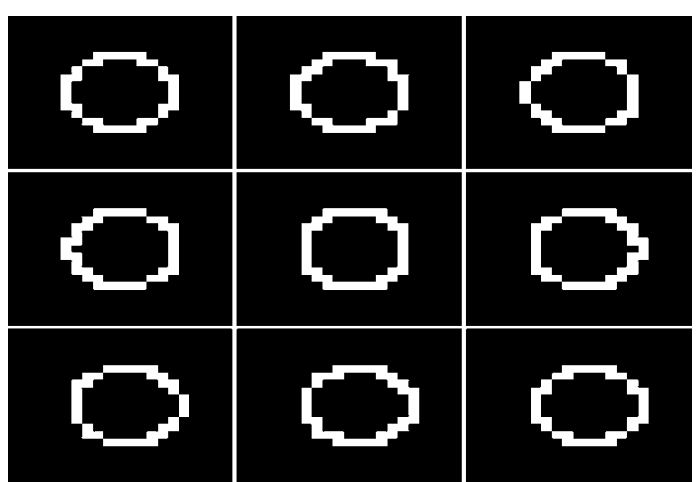

a Corresponding author: fabrice.mairesse@u-bourgogne.fr 
De plus, les formes à étudier, le plus souvent des usinages, ne sont eux même pas des formes parfaitement géométriques. Plus particulièrement, le contour d'un perçage ne sera pas circulaire ne serait-ce qu'à cause des résidus de matière. Enfin, les outils de segmentation d'images visant à extraire cet usinage de son environnement peuvent fournir un contour de mauvaise qualité voire partiel. Il ne s'agira donc pas de déterminer les paramètres d'un cercle mais plutôt d'estimer, à partir d'un ensemble de pixels, le cercle qui correspond le mieux.

Une approche de correction de forme visant l'amélioration de la mesure sera proposée en section 2 . La section 3 présentera des algorithmes permettant d'estimer le rayon et la position du centre d'un cercle. Une étude expérimentale combinant correction de forme et estimateurs sera menée dans la section 4. La conclusion sera donnée en section 5 .

\section{Correction de formes circulaires}

Il existe de nombreuses méthodes pour approximer les formes. Parmi celles-ci, on trouve les méthodes de contours actifs qui se trouvent être bien adaptées aux formes circulaires. En effet, elles se basent sur des principes de minimisation d'énergie interne et externe et permettent d'obtenir un contour continu en conservant une certaine régularité, une tolérance au bruit, etc. selon la formulation choisie. C'est pourquoi les contours actifs sont communément utilisés pour la régularisation de formes en général.

Cependant, il demeure une limite notable à ces techniques. Ces dernières se limitent à une représentation unique du contour obtenue après un certain nombre de cycles dépendant notamment de l'image et des conditions initiales. Il est possible d'obtenir d'autres représentations intermédiaires en limitant le nombre de cycles mais pour des résultats souvent peu pertinents. Ceci oblige à effectuer de multiples exécutions avec différents paramètres, ce qui est rarement compatible avec le temps de contrôle disponible en contrôle industriel.

Afin d'obtenir un comportement proche des contours actifs mais permettant d'obtenir une multiple représentation du contour en une seule exécution, les auteurs ont considéré la propagation de fronts d'ondes dont la hauteur de transition permet de définir un niveau de régularité. De plus, une attention particulière a été portée aux notions de connexité notamment seconde, présentes en morphologie mathématique [1-4].

Le comportement de ce contour actif est proche de la dilatation géodésique $[1,2]$ et utilisera donc la notion de marqueur. Soient A, un marqueur (Fig. 2) d'où partira la propagation et $\mathrm{B}$ une contrainte topologique issue de l'image (Fig. 2). Le couple (A, B) de fonctions discrètes scalaires définit l'équation :

$$
E_{e}(A, B):\left\{\begin{array}{c}
\frac{d v_{n, m}}{d t}=\frac{D(B)}{e}\left(v_{n-1, m}+v_{n+1, m}+v_{n, m-1}\right. \\
\left.+v_{n, m+1}-4 \cdot v_{n, m}\right)-f_{a}\left(v_{n, m}\right) \\
\operatorname{avec} f_{a}(v)=v(a-v)(1-v) \text { pour } a<\frac{1}{2}, \\
D(B)=\frac{1+\tanh (20 . B-12)}{2}, \text { et }\left.v\right|_{t=0}=A
\end{array}\right\}
$$

Le système est complété par les conditions de Neumann aux limites du domaine. Le marqueur demeure constant et égal à 1 et seule la propagation de l'état 1 est stable du fait de a pris inférieur à 0.5 (Fig. 2).

La propagation est contrôlée par le paramètre de diffusion D. Si $\mathrm{D}<\mathrm{D}_{\text {critique }}<<1$, la propagation s'arrête. De fait, la propagation est autorisée si la longueur d'onde du front est suffisamment petite pour passer, connectant ou non les pixels. Ainsi, une représentation multi-échelle est obtenue (Fig. 2).

La figure 3 illustre la décomposition multi-échelle du contour actif. Selon la valeur de seuillage, on obtient une représentation plus ou moins approchée des formes initiales. Plus le seuil est haut, plus les formes sont approchées.

Figure 2. haut gauche : marqueurs, haut droite : image initiale bas : propagation du contour actif

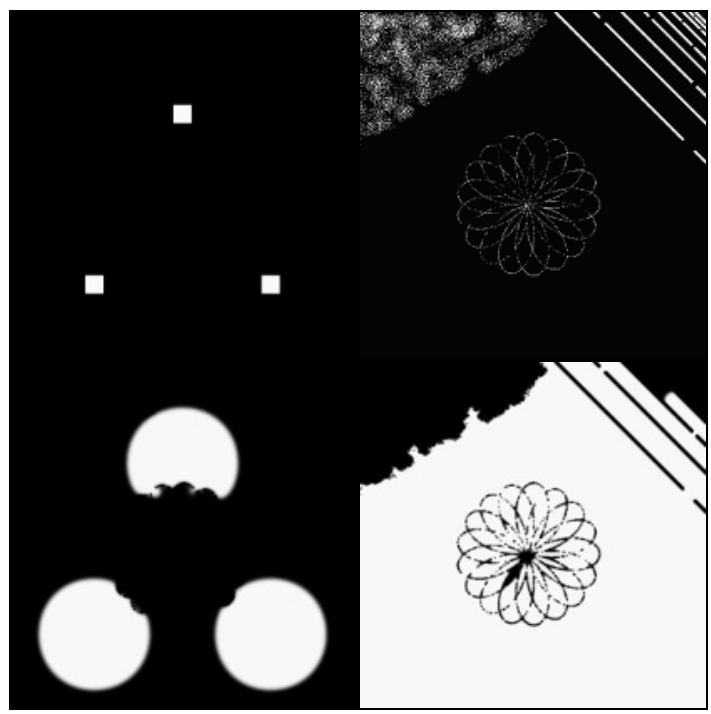


Figure 3. Différentes représentations par seuillage

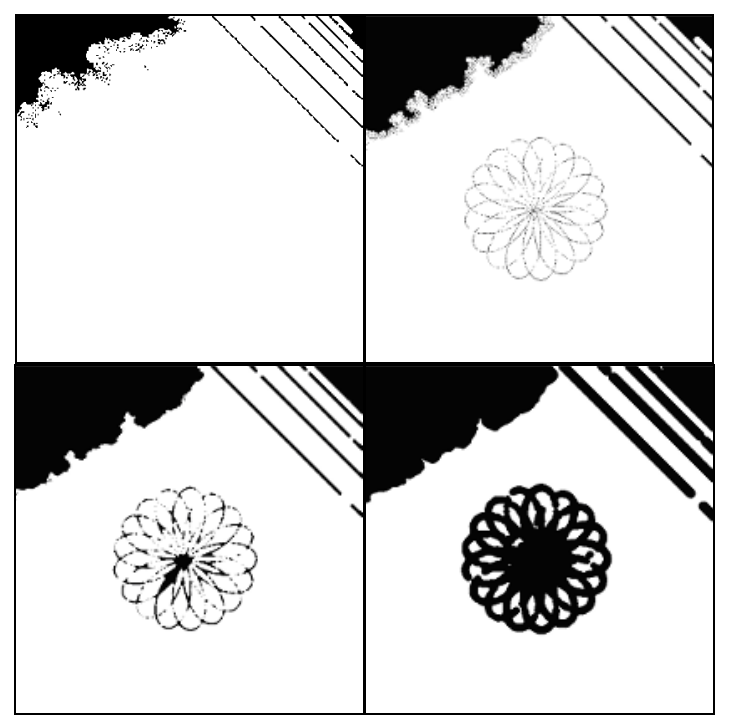

\section{Estimateurs de cercles}

\subsection{Estimation par barycentre}

L'estimateur le plus connu est sans doute le barycentre. Son application au domaine du traitement d'images est directe puisqu'il suffit de prendre pour poids les valeurs des pixels du contour considéré. On obtient ainsi une estimation de la position du centre du cercle.

\subsection{Estimation par moindres carrés}

Aussi connu que le barycentre, les estimateurs par moindres carrés permettent d'accéder à la position du centre et au rayon du cercle. Très rapide, ils sont souvent utilisés en vision industrielle. De nombreuses implémentations sont possibles et Les auteurs ont implémenté l'algorithme proposé dans [5,6].

\subsection{Estimation par Hough}

Enfin la dernière méthode classique repose sur la transformée de Hough circulaire, également appelée transformée de Hough 3D [7]. Elle repose sur un principe d'accumulation de votes [8]. Elle est généralement peu utilisée en industrie du fait de son coût de calculs élevé. La méthode présentée ici intègre une seconde phase d'estimation permettant d'obtenir des valeurs réelles par fitting de l'estimateur [9].

\subsection{Estimation par Radon}

La transformée de Radon est un outil fréquent pour la detection de lignes. Elle est robuste au bruit et fournit toutes les lignes présentes dans une image. Elle est proche de la transformée de Hough et utilise également un accumulateur de votes. La transformée de Radon est donnée par l'équation :

$$
\begin{gathered}
R_{\theta}\left(x^{\prime}\right)=\int_{-\infty}^{+\infty} f\left(x^{\prime} \cos \theta-y^{\prime} \sin \theta, x^{\prime} \sin \theta+y^{\prime} \cos \theta\right) d y^{\prime} \\
\text { avec }\left[x^{\prime}, y^{\prime}\right]=\left[\begin{array}{cc}
\cos \theta & \sin \theta \\
-\sin \theta & \cos \theta
\end{array}\right]\left[\begin{array}{l}
x \\
y
\end{array}\right] \text { et } f \text { l'image }
\end{gathered}
$$

L'utilisation de cette méthode n'est a priori pas adaptée pour l'estimation de cercle. L'idée est ici de déterminer les tangentes au cercle et d'accéder à la position des rayons. Ceci permet de déterminer leurs intersections i.e. le centre du cercle.

Si l'on considère la représentation d'un cercle sur une grille discrète, on obtient un ensemble de segments. Il est donc possible de les trouver à l'aide de la transformée de Radon. De même, bien qu'une tangente ne passe mathématiquement que par un point, ce n'est plus le cas ici du fait de la discrétisation. Une tangente discrète passera donc par un ou plusieurs pixels. C'est cette propriété qui sera utilisée ici [10].

De l'équation (2), on constate que la transformée de Radon permet le passage d'une représentation cartésienne de l'image vers une représentation polaire de celle-ci. Chaque droite se retrouve donc définie par des paramètres $\rho$ et $\theta$. La valeur au point $\left(\rho_{1}, \theta_{1}\right)$ correspond au nombre de pixels alignés selon la ligne $\mathrm{L}_{1}$. Ainsi plus la valeur est élevée, plus la ligne est probable dans l'image.

La recherche des maxima conduit donc à trouver les tangentes discrètes au cercle. La distance entre deux tangentes parallèles est théoriquement de deux fois le rayon. On en déduit les droites portant les rayons et donc le centre du cercle. Les auteurs sont conscients que ces tangentes discrètes ne correspondent pas aux tangentes théoriques mais peuvent être vues comme des représentations proches et épaisses.

Soit le point de coordonnées (x,y). Dans le domaine de Radon, ce point se caractérise par une courbe ayant pour équation :

$$
d=x \cos (o)-y \sin (o)
$$

L'intersection des rayons étant un point, il doit donc respecter l'équation (3). En procédant à un ajustement analytique, il est donc possible de remonter aux valeurs réelles de $\mathrm{x}$ et $\mathrm{y}$. L'ajustement considéré ici est basé sur un algorithme à régions de confiance par moindres carrés non linéaires [11] combiné à une élimination des points aberrants de type $\alpha . \sigma$.

\section{Etude expérimentale}

Afin d'étudier les incertitudes d'estimations sur la position du centre et le rayon d'un cercle, un ensemble de contours de cercles déformés a été générée. Ces cercles ne feront apparaître que des déformations intérieures, les rayons allant de 20 à 100 pixels par pas de 5 pixels. Pour chaque rayon, vingt cercles seront générés avec un centre à valeurs réelles, soit un total de 340 cercles. 


\subsection{Modèle de cercles imparfaits}

Les cercles sont décrits par l'équation suivante :

$$
\begin{aligned}
& \left\{x=E\left[x_{0}+r \cos (\theta)\right], y=E\left[y_{0}+r \sin (\theta)\right]\right\} . \\
& r=R-\max \left(0, r_{\text {distortion }}\right), R \text { le rayon du cercle idéal } \\
& r_{\text {distortion }}=a_{0}+\sum_{k=1}^{50} a(k) \cos (k \theta)+b(k) \sin (i \theta) . \\
& a_{0} \text { aléatoire à valeur dans }\left[0, \frac{R}{10}\right], \\
& a(i)=a_{\sigma}\left(\frac{\frac{R}{10}}{0.8+\left(\frac{i}{5}\right)^{p_{\sigma}}}\right), b(i)=b_{\sigma}\left(\frac{\frac{R}{10}}{0.8+\left(\frac{i}{5}\right)^{p_{\sigma}}}\right) . \\
& \begin{array}{l}
a_{\sigma}, b_{\sigma} \text { et } p_{\sigma}, \text { variables aléatoires } \\
\text { normalem ent distribuées. }
\end{array}
\end{aligned}
$$

Cette formulation permet d'obtenir des cercles présentant des zones intactes et des zones faiblement ou fortement modifiées (Fig. 4).

Figure 4. Exemples de cercles générés.

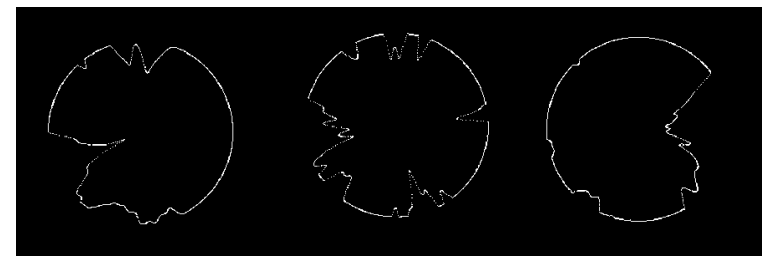

L'application d'une correction de formes (éq. (1)) permet de régulariser les contours et d'atténuer plus ou moins les imperfections selon l'échelle choisie (Fig. 5).

Figure 5. Contour actif final et formes régularisées.

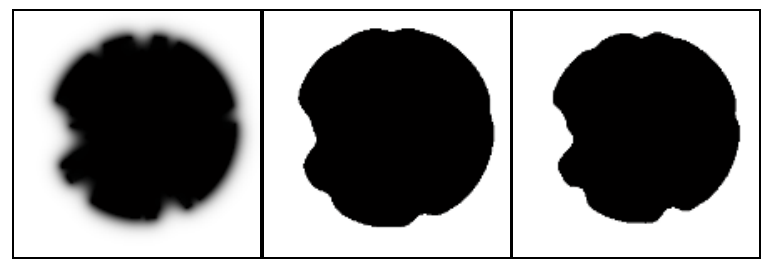

\subsection{Résultats}

Afin de quantifier l'erreur faite dans l'estimation de la position du centre du cercle, la distance euclidienne entre la position théorique et estimée est calculée. La moyenne et l'incertitude type de ces distances est faite selon le diamètre théorique du cercle. Pour le rayon, la moyenne et l'incertitude type de la différence absolue entre le rayon théorique et le rayon estimé est également faite. Les résultats se trouvent résumés dans les tables 1 et 2 .

Dans un souci de simplification de lecture, une représentation graphique de ces données est disponible (fig. 6, 7, 8 et 9). Elles proposent les résultats des méthodes avec correction de formes ainsi que la différence avec les formes non corrigées.

\subsubsection{Barycentre}

L'estimateur par barycentre est un bon estimateur de centre de cercle déformé. L'erreur moyenne maximale est de 3.83 pixels $(\sigma=2.66$ pixels $)$ et l'erreur moyenne minimale de 1.25 pixels $(\sigma=0.64$ pixel). Elle tend légèrement à augmenter avec l'augmentation du rayon. Un prétraitement de la forme ne semble pas affecter de façon notable le résultat bien qu'une légère amélioration globale est perceptible avec une erreur moyenne maximale de 3.03 pixels à $(\mathrm{r}=100$ pixels, $\sigma=2.46$ pixels) et minimale de 1.28 pixels $(r=20$ pixels, $\sigma=0.69$ pixel).

\subsubsection{Moindres carrés}

L'estimateur par moindres carrés apparaît comme inadapté à la mesure de cercle imparfait. En effet, l'erreur moyenne ne cesse d'augmenter avec le rayon allant de 1.84 pixel $(\mathrm{r}=20$ pixels, $\sigma=0.99$ pixel $)$ à 7.11 pixels $(\mathrm{r}=$ 100 pixels, $\sigma=3.09$ pixels). La régularisation de la forme a ici une influence forte puisqu'on constate une diminution de l'erreur moyenne de 0.5 à 1.8 pixel et de l'incertitude type de 0.4 à 0.8 pixel.

L'estimation du rayon suit la même évolution avec un impact de la régularisation encore plus notable puisqu'elle passe de $[2.08,10.68]$ à $[0.54,1.85]$ avec un abaissement de l'incertitude type.

\subsubsection{Hough $3 D$}

L'étude des résultats de l'estimateur par transformée de Hough est plus complexe. Il est ainsi pertinent pour des rayons supérieurs à 40 pixels mais relativement mauvais en dessous tout en conservant une incertitude type faible et stable. Ceci s'explique par le principe de votes qui augmentent avec le rayon. L'approximation des cercles augmente légèrement l'erreur moyenne et l'incertitude type. Ceci est dû aux modifications du contour global impactant le processus d'accumulation de votes. Son impact est plus notable au niveau de l'estimation du rayon où l'on voit diminuer l'erreur moyenne pour un rayon inférieur à 60 pixels à incertitude type comparable. Au-delà, le contour actif nuit à l'estimation.

\subsubsection{Radon}

L'estimateur par transformée de Radon non corrigée est clairement un mauvais estimateur puisque l'erreur moyenne évolue entre 4 et 19 pixels et une incertitude type élevée. Cet estimateur ne donne des résultats convaincants que combiné à une correction de formes. L'erreur moyenne va alors de $1.05(\mathrm{r}=20$ pixels, $\sigma=$ 0.48 pixel) à 9.02 pixels $(\mathrm{r}=100$ pixels, $\sigma=5.24$ pixel $)$. Les mêmes constatations peuvent être faites concernant l'estimation du rayon. Ceci en fait un estimateur performant s'il reste limité aux cercles de faibles rayons [9]. 
Table 1. Erreur moyenne et incertitude type des estimateurs de cercle pour la position du centre.

\begin{tabular}{|c|c|c|c|c|c|c|c|c|c|c|c|c|c|c|c|c|c|c|c|}
\hline Méthode & Correction & Rayon & 20 & 25 & 30 & 35 & 40 & 45 & 50 & 55 & 60 & 65 & 70 & 75 & 80 & 85 & 90 & 95 & 100 \\
\hline \multirow{4}{*}{ Barycentre } & \multirow[b]{2}{*}{ sans } & erreur moyenne & 1,34 & 1,25 & 1,78 & 1,44 & 2,50 & 2,07 & 2,32 & 2,18 & 2,10 & 2,50 & 2,19 & 3,55 & 3,02 & 3,18 & 3,50 & 3,83 & 2,92 \\
\hline & & incertitude type & 0,55 & 0,64 & 1,07 & 0,91 & 1,41 & 1,07 & 1,06 & 1,82 & 1,68 & 1,38 & 1,35 & 2,78 & 2,19 & 2,02 & 2,94 & 2,66 & 1,88 \\
\hline & \multirow{2}{*}{ avec } & erreur moyenne & 1,28 & 1,29 & 1,72 & 1,35 & 2,13 & 1,78 & 1,68 & 1,73 & 2,02 & 1,88 & 2,27 & 3,02 & 2,76 & 3,03 & 2,41 & 2,84 & 3,03 \\
\hline & & incertitude type & 0,69 & 0,62 & 1,24 & 0,79 & 1,62 & 0,94 & 1,28 & 1,27 & 1,17 & 1,19 & 1,45 & 2,96 & 2,36 & 1,20 & 2,68 & 1,97 & 2,47 \\
\hline \multirow{4}{*}{$\begin{array}{c}\text { Moindres } \\
\text { carrés }\end{array}$} & \multirow{2}{*}{ sans } & erreur moyenne & 1,84 & 2,24 & 2,92 & 2,48 & 3,92 & 3,79 & 4,05 & 4,00 & 3,99 & 3,94 & 5,52 & 6,49 & 6,63 & 6,67 & 5,60 & 6,72 & 7,11 \\
\hline & & incertitude type & 0,99 & 1,05 & 1,88 & 1,10 & 2,23 & 1,47 & 2,12 & 2,46 & 2,32 & 2,24 & 2,66 & 4,65 & 3,75 & 3,64 & 4,20 & 4,08 & 3,09 \\
\hline & \multirow[b]{2}{*}{ avec } & erreur moyenne & 1,35 & 1,54 & 2,07 & 1,73 & 2,74 & 2,59 & 2,91 & 2,77 & 2,80 & 2,82 & 3,78 & 4,78 & 4,90 & 4,86 & 4,41 & 5,12 & 5,34 \\
\hline & & incertitude type & 0,69 & 0,75 & 1,39 & 0,86 & 1,80 & 0,98 & 1,52 & 1,92 & 1,87 & 1,64 & 2,22 & 3,96 & 2,93 & 2,64 & 3,44 & 3,35 & 2,25 \\
\hline \multirow{4}{*}{ Hough 3D } & \multirow{2}{*}{ sans } & erreur moyenne & 3,96 & 3,47 & 2,59 & 2,41 & 1,91 & 1,76 & 1,82 & 1,57 & 1,22 & 1,23 & 1,18 & 1,16 & 0,98 & 1,09 & 0,90 & 0,72 & 0,86 \\
\hline & & incertitude type & 0,59 & 0,62 & 0,44 & 0,56 & 0,30 & 0,21 & 0,41 & 0,39 & 0,42 & 0,39 & 0,44 & 0,46 & 0,42 & 0,34 & 0,24 & 0,28 & 0,37 \\
\hline & \multirow{2}{*}{ avec } & erreur moyenne & 2,92 & 2,95 & 2,77 & 2,71 & 2,41 & 2,23 & 1,90 & 1,99 & 1,42 & 1,48 & 1,45 & 1,31 & 1,16 & 1,07 & 1,08 & 0,90 & 0,94 \\
\hline & & incertitude type & 0,62 & 0,90 & 0,62 & 0,58 & 0,73 & 0,77 & 0,59 & 0,49 & 0,53 & 0,65 & 0,60 & 0,81 & 0,63 & 0,57 & 0,58 & 0,64 & 0,65 \\
\hline \multirow{4}{*}{ Radon } & \multirow{2}{*}{ sans } & erreur moyenne & 4,05 & 5,97 & 6,03 & 7,13 & 9,39 & 9,87 & 11,44 & 10,36 & 7,38 & 12,40 & 13,90 & 17,25 & 16,08 & 17,01 & 14,31 & 17,33 & 19,10 \\
\hline & & incertitude type & 2,39 & 2,67 & 3,04 & 3,66 & 5,31 & 3,98 & 5,32 & 7,71 & 6,67 & 6,91 & 5,90 & 11,45 & 9,79 & 11,11 & 12,44 & 13,46 & 10,06 \\
\hline & \multirow{2}{*}{ avec } & erreur moyenne & 1,05 & 1,19 & 1,81 & 2,08 & 3,95 & 4,23 & 5,20 & 4,68 & 4,38 & 5,24 & 6,28 & 7,80 & 8,83 & 9,19 & 7,93 & 8,74 & 9,02 \\
\hline & & incertitude type & 0,48 & 0,74 & 1,44 & 2,27 & 2,91 & 1,79 & 3,27 & 3,55 & 4,21 & 3,52 & 4,34 & 6,81 & 7,32 & 8,21 & 7,15 & 6,58 & 5,24 \\
\hline
\end{tabular}

Table 1. Erreur moyenne et incertitude type des estimateurs de cercle pour le diamètre.

\begin{tabular}{|c|c|c|c|c|c|c|c|c|c|c|c|c|c|c|c|c|c|c|c|}
\hline Méthode & Correction & Rayon & 20 & 25 & 30 & 35 & 40 & 45 & 50 & 55 & 60 & 65 & 70 & 75 & 80 & 85 & 90 & 95 & 100 \\
\hline \multirow{4}{*}{$\begin{array}{c}\text { Moindres } \\
\text { carrés }\end{array}$} & \multirow[b]{2}{*}{ sans } & erreur moyenne & 2,08 & 2,87 & 3,08 & 3,74 & 4,94 & 5,19 & 5,98 & 5,95 & 5,37 & 7,33 & 7,63 & 9,01 & 8,00 & 9,25 & 10,01 & 9,85 & 10,68 \\
\hline & & incertitude type & 0,99 & 1,05 & 1,88 & 1,10 & 2,23 & 1,47 & 2,12 & 2,46 & 2,32 & 2,24 & 2,66 & 4,65 & 3,75 & 3,64 & 4,20 & 4,08 & 3,09 \\
\hline & \multirow[b]{2}{*}{ avec } & erreur moyenne & 1,46 & 1,00 & 0,91 & 0,92 & 0,89 & 0,54 & $\begin{array}{l}0,96 \\
\end{array}$ & 0,90 & 1,00 & 1,50 & 1,22 & 1,69 & 1,75 & 1,41 & 1,77 & 1,83 & 1,85 \\
\hline & & incertitude type & 0,39 & 0,54 & 0,59 & 0,55 & 0,73 & 0,41 & 0,86 & 0,81 & 0,70 & 1,15 & 0,57 & 1,38 & 1,14 & 1,46 & 1,28 & 1,68 & 1,59 \\
\hline \multirow{4}{*}{ Hough 3D } & \multirow{2}{*}{ sans } & erreur moyenne & 3,76 & 3,10 & 2,33 & 2,08 & 1,71 & 1,48 & 1,36 & 1,24 & 1,01 & 0,99 & 0,91 & 0,85 & 0,74 & 0,71 & 0,67 & 0,59 & 0,62 \\
\hline & & incertitude type & 0,59 & 0,62 & 0,44 & 0,56 & 0,30 & 0,21 & 0,41 & 0,39 & 0,42 & 0,39 & 0,44 & 0,46 & \begin{tabular}{l|l}
0,42 \\
\end{tabular} & 0,34 & 0,24 & 0,28 & 0,37 \\
\hline & \multirow{2}{*}{ avec } & erreur moyenne & 1,30 & 0,75 & 0,39 & 0,30 & 0,25 & 0,38 & 0,53 & 0,73 & 1,12 & 1,06 & 1,22 & 1,26 & 1,53 & 1,43 & 1,69 & 1,89 & 1,79 \\
\hline & & incertitude type & 0,33 & 0,43 & 0,39 & 0,27 & 0,22 & 0,18 & 0,21 & 0,35 & \begin{tabular}{l|l}
0,46 \\
\end{tabular} & 0,36 & 0,51 & 0,45 & 0,49 & 0,51 & 0,52 & 0,50 & 0,55 \\
\hline \multirow{4}{*}{ Radon } & \multirow{2}{*}{ sans } & erreur moyenne & 4,07 & 5,84 & 5,38 & 6,82 & 8,49 & 8,93 & 10,88 & 9,90 & 7,69 & 11,53 & 12,71 & 15,14 & 13,68 & 14,53 & 15,04 & 15,44 & 17,04 \\
\hline & & incertitude type & 2,39 & 3,19 & 3,29 & 3,66 & 5,27 & 5,14 & 6,08 & 7,71 & 6,67 & 7,81 & 6,64 & 11,45 & 9,79 & 11,22 & 12,44 & 13,46 & 12,20 \\
\hline & \multirow{2}{*}{ avec } & erreur moyenne & 0,74 & 0,69 & 1,23 & 1,79 & 1,62 & 1,08 & 1,67 & 1,59 & 2,08 & 2,53 & 2,13 & 3,35 & 4,02 & 3,12 & 3,11 & 4,00 & 3,32 \\
\hline & & incertitude type & 0,36 & 0,63 & 0,70 & 1,74 & 1,15 & 0,83 & 1,88 & 1,28 & 1,89 & 1,74 & 1,53 & 2,26 & 2,28 & 3,62 & 2,61 & 3,65 & 2,83 \\
\hline
\end{tabular}

Figure 6. Erreur moyenne de l'estimation du centre.

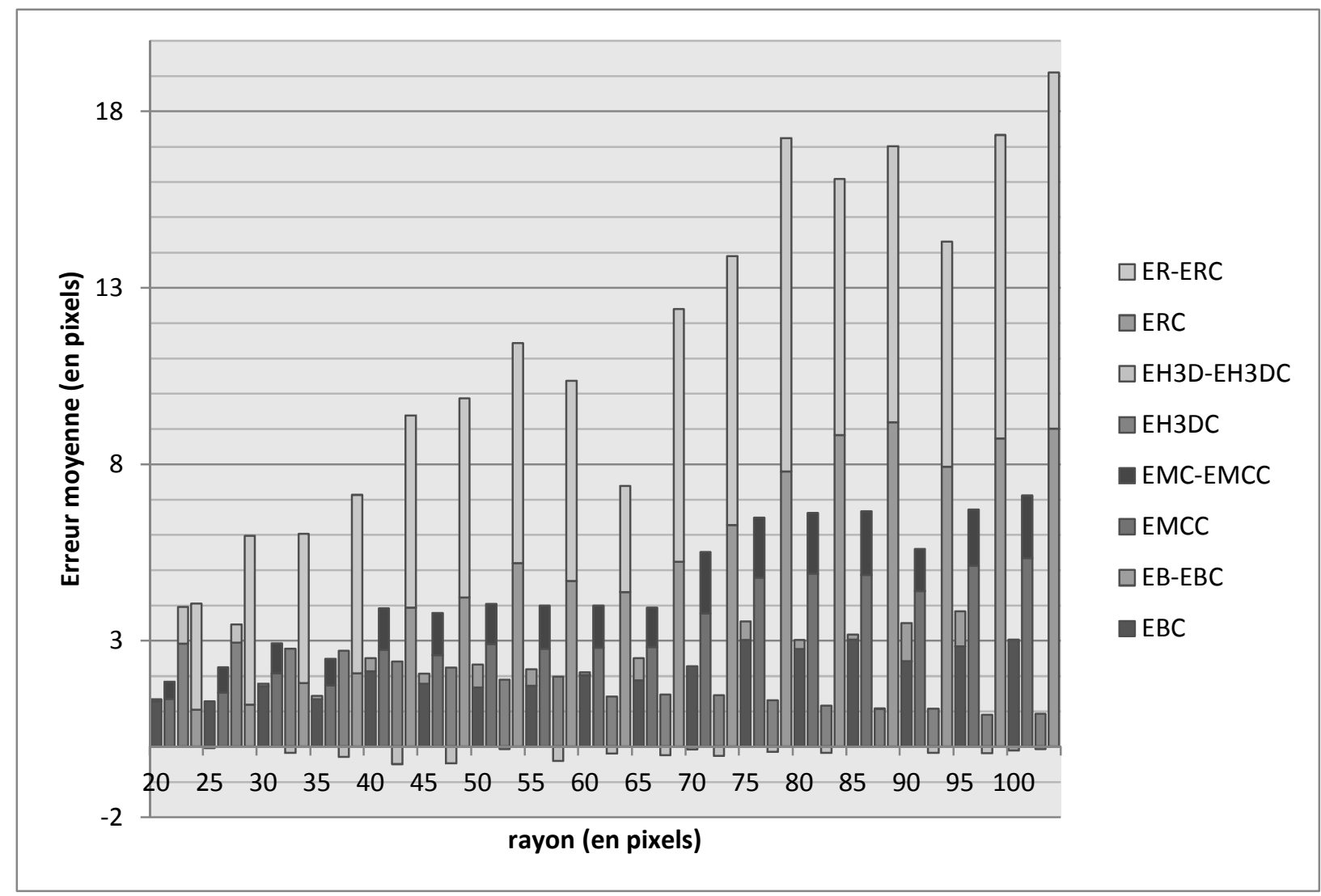

$\overline{{ }^{a}}$ Corresponding author: fabrice.mairesse@u-bourgogne.fr 
Figure 7. Incertitude type de l'estimation du centre.

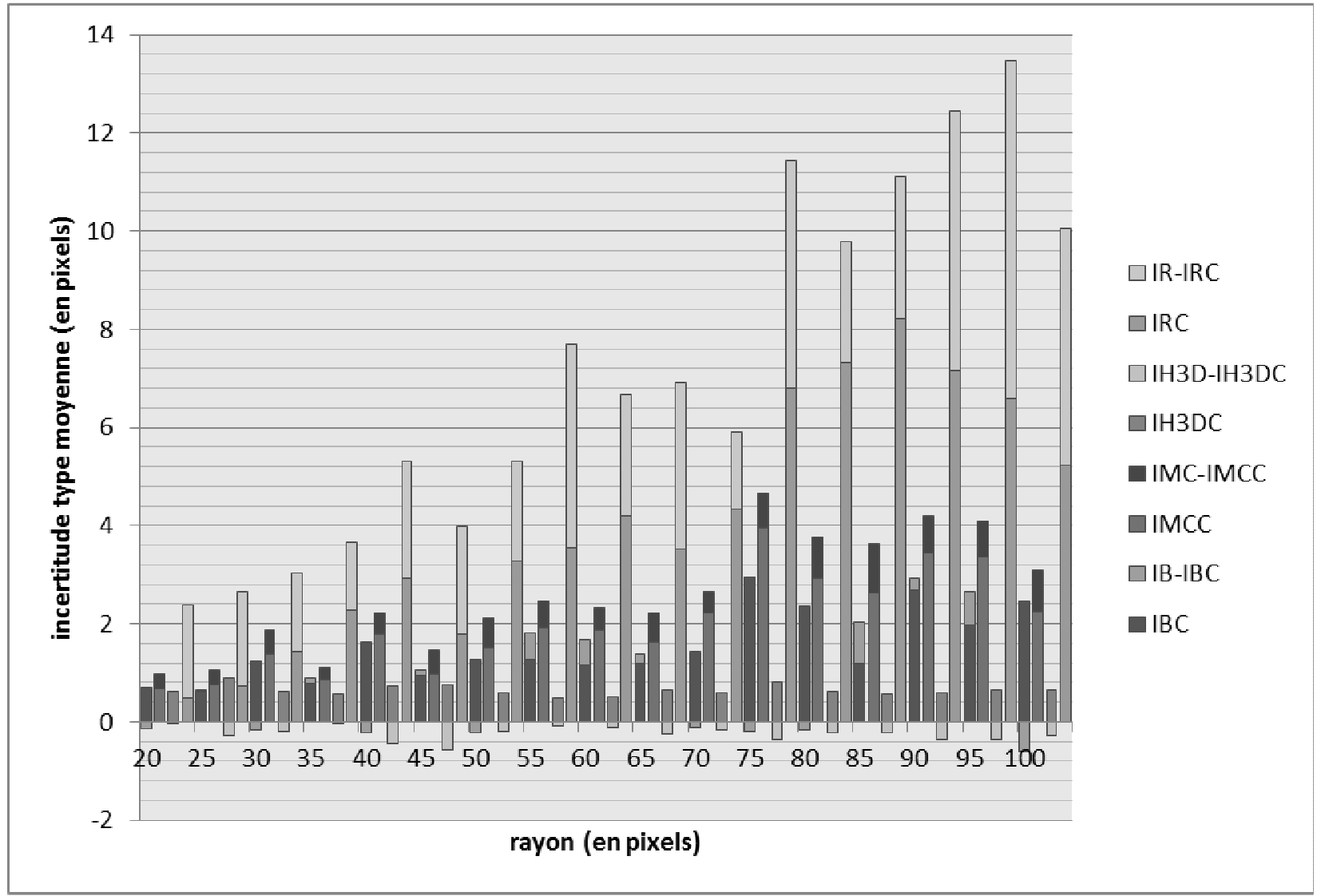

Figure 8. Erreur moyenne de l'estimation du rayon.

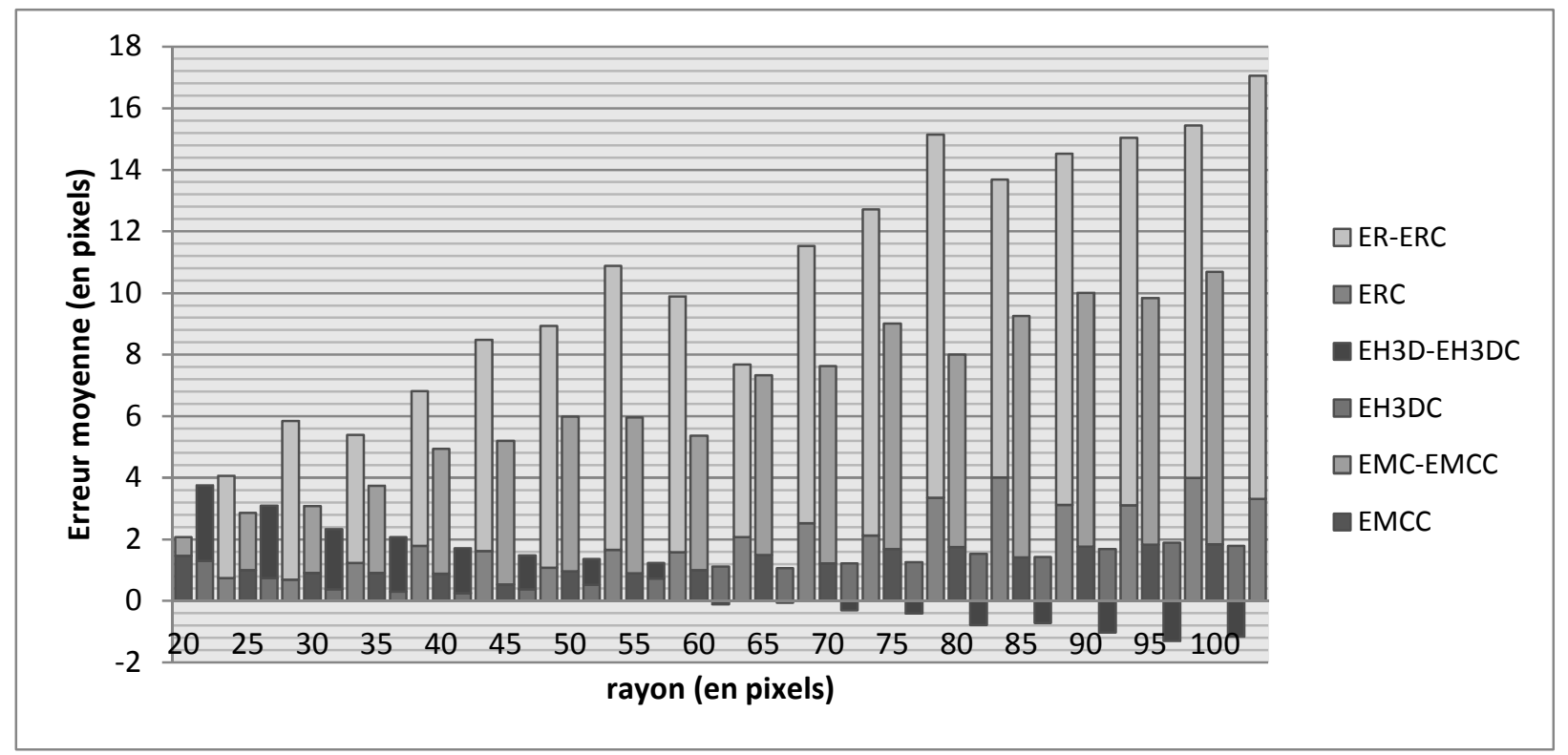


Figure 9. Incertitude type de l'estimation du rayon.

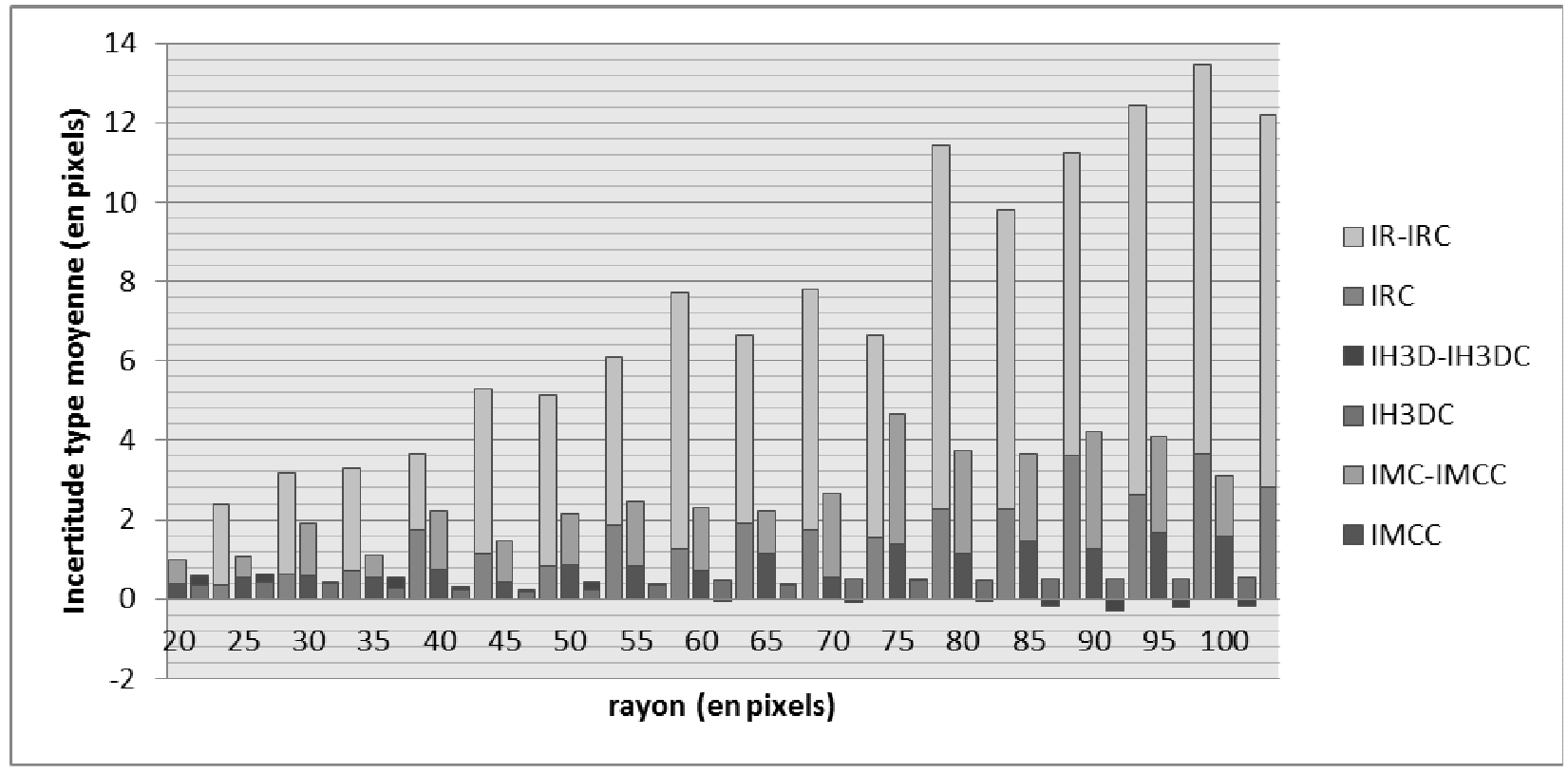

\section{Conclusion}

Dans cette étude, les auteurs proposent plusieurs outils pour le mesurage des caractéristiques d'un cercle. Ces techniques sont testées dans le cadre de cercles déformés. De plus, une méthode de régularisation de formes est proposée afin d'améliorer la mesure. Cette approche est intéressante pour deux des estimateurs (moindre carrés et transformée de Radon qui voient leurs erreur moyenne et incertitude type clairement diminuées. Le résultat demeure plus nuancé pour le barycentre et la transformée de Hough 3D. L'estimation par barycentre n'est que peu améliorée par la régularisation. Dans le cas de la transformée de Hough 3D, l'approximation de formes par contours actifs n'a d'intérêt que pour les cercles de rayons inférieurs à 40 pixels. Au-delà, la modification du contour nuit au principe d'accumulation utilisé servant à l'estimation.

La méthode de contours actifs présentée peut être implémentée électroniquement, permettant ainsi le calcul des approximations de formes à grande vitesse [12]. Les résultats sur d'autres types de déformations ou bruit sont également disponibles dans [9]

\section{Références}

1. J. Serra, Image Analysis and Mathematical Morphology Vol. I., Academic Press, London, 1982

2. J. Serra, Image Analysis and Mathematical Morphology Vol. II: Theoretical Advances, Academic Press, London, 1988
3. R. Goldenberg, R. Kimmel, E. Rivlin, M. Rudzsky, "Fast Geodesic Active Contours", IEEE Trans. Image Proc., 10(10), (2001)

4. N. Paragios, R. Deriche, "Geodesic Active Contours and Level Sets for the Detection and Tracking of Moving Objects", IEEE Trans. PAMI, 22(3), (2000)

5. W. Gander, G.H. Golub, R. Strebel, "Least-squares fitting of circles and ellipses", BIT, 34, (1994)

6. N. Chernov, C. Lesort, "least-squares fitting of circles and lines", Computer vision and pattern recognition, (2003)

7. D.S Luo, P. Smart, J.E.S. Macleod, "Circular hough transform for roundness measurement of objects", Pattern recognition, 28(11), (1995)

8. S. Chiu, J. Liaw, "An effective voting method for circle detection", Pattern recognition letters, 26(2), (2005)

9. F. Mairesse, T. Sliwa, S. Binczak, Y. Voisin, "Subpixel determination of imperfect circles characteristics", Elsevier Pattern Recognition, 41 (1), 2008

10. H. Kim, J. Kim, “A two-step circle detection algorithm from the intersecting chords", Pattern recognition letters, 22, (2001)

11. M.A. Branch, T.F. Coleman, Y. Li, "A subspace, interior, and conjugate gradient method for largescale bound-constrained minimization problems", SIAM Journal of scientific computing, 21(1), (1999)

12. S. Binczak, T. Sliwa, S. Jacquir, J.-M. Bilbault, "Reaction-Diffusion Network For Geometric Multiscale High Speed Image Processing", Image and Vision Computing, 28, 2010 